\section{The Systems Biology of Stem Cell Released Molecules: A Novel Approach to Optimizing Animal Health}

\section{Joel Ehrenzweig ${ }^{1 *}$, Peter Friedman ${ }^{2}$ and Anthony Martin ${ }^{3}$}

\begin{abstract}
Veterinary drug and therapeutic development has traditionally been based on the theory of "reductionism", when (1) a clinical state is broken down to a defined biochemical pathway underlying the condition; (2) a target in the pathway is identified; (3) a drug interacting with the target is developed; and (4) the target is modified to ameliorate the disease. But biological systems are extremely complex, and the reductionist approach to developing therapeutic solutions is limited: Diseased or traumatized tissue frequently involve multiple underlying pathways. True mitigation of a disease process that fully allows the patient healing requires an approach that is multifaceted and systems-based.

A resolution to this reductionist conundrum may be found in the use of stem cell based therapeutics. Significant data explains how stem cells repair and regenerate tissue and modulate the immune system: As much as $80 \%$ of the beneficial effects of stem cells result from their ability to release a multitude of molecules. These stem cell released molecules (SRM) modulate the cellular milieu to evoke a multitude of responses from neighboring cells. Stem cells represent a natural, systems-based biological 'factory', producing and releasing a host of molecules capable of interacting with the system of biomolecular circuits underlying a variety of indications. Current research includes efforts to define, stimulate, enhance and harness SRM, facilitating the development of new topical products and systems-therapeutics. Heretofore, advances in the development of SRM-based products have focused exclusively on human health applications. This paper discusses the translational potential and significant promise of repurposed existing human drugs and therapeutics and their application into companion animal treatments.
\end{abstract}

\section{Keywords}

Stem cells: Animal Health; Disease; Canine

\section{Introduction}

Billions of dollars have been spent by human pharmaceutical companies that continue to rely upon the reductionist approach in their search for new, single entity drugs, many based upon the expectation that genomic data can identify possible drug candidates. However, in our current post genomic era [1], knowing the sequence of the genome has limited predictive value in disease diagnosis and

*Corresponding author: Joel Ehrenzweig, BS, DVM, Consultant, TWH Consulting LLC, 1619 Denby Way, Midlothian Virginia 23114 USA, Tel: +1.804.432.5664; Fax: +1.804.482.2898; E-mail: petvet@hotmail.com

Received: May 22, 2017 Accepted: June 09, 2017 Published: June 13, 2017 treatment $[2,3]$. Thus pharmaceutical companies are beginning to realize that new diagnostic and therapeutic regimens are needed beyond those that rely on simple genomics [4]. While research and development costs in the pharmaceutical industry continue to increase, the number of newly approved drugs is on a steady decline, and new paradigms for drug development are being proffered [5-7]. Following the rapid emergence of in vitro and in silico screenings, including molecular and genetic tools, now there are advances in systems-based means necessary to describe the effects of drug candidates within the complex biochemical pathways of intact, fully assembled living networks [8-11]. The pharmaceutical industry is retreating from its overreliance on non-predictive genetic tools [4] and focusing instead on systems approach. Big Pharma has accepted the need for innovative strategies and technologies so that new drug candidates can be identified and developed.

\section{Systems Based SRM Therapeutics}

Rather than reliance on a traditional utilization of narrow reductionist methodology that locks single molecule development into a constricted target pathway, a system-based therapeutic approach is expansive.

The systems approach relies on an understanding and appreciation of a cellular milieu within which stem cells are actively contributing to their environment. Desired therapeutic results are achieved by the release of SRM consisting of multiple cytokines, growth factors, extracellular matrix (ECM) molecules, micro-RNA, antioxidants and other molecules that act either on themselves (autocrine actions) or on neighboring cells (paracrine actions) (Figure 1). The SRM pathways are significantly more robust compared to the route where differentiation is the key mechanism for tissue repair. This is further discussed herein. Additionally, adult stem cells are recognized to be involved in the daily maintenance, regeneration and repair of tissue and are likely involved in immune system functions and fighting infection [12,13].

A clear understanding of stem and progenitor cell biomolecule production may: (1) yield new insights into the regulation of cell phenotypes; (2) better define the functional role of stem cells in tissue maintenance, replication and repair processes; and (3) better determine appropriate cell sources for specific tissue repair and regenerative applications. The ultimate goal is the development of new drugs, biologics and therapeutics. Current research directions include efforts to define, stimulate, enhance and harness the SRM and their autocrine and paracrine mechanisms for regenerative medicine and therapeutic development.

The power of the SRM system versus reductionist therapeutics has been reported by Eppler et al. [14]. It was found that a single component of intravenously administered SRM, vascular endothelial growth factor (VEGF) with a 30-minute half-life, undergoes rapid degradation through denaturation, oxidation and proteolysis [15]. However, when SRM that contain VEGF are administered in a collection that includes extracellular chaperones and antioxidants rather than a single molecule, then the resultant half-life is greatly extended [16-22].

A dramatic example illustrating SRM wound healing power,
ScîTechnol Technology and Medicine 


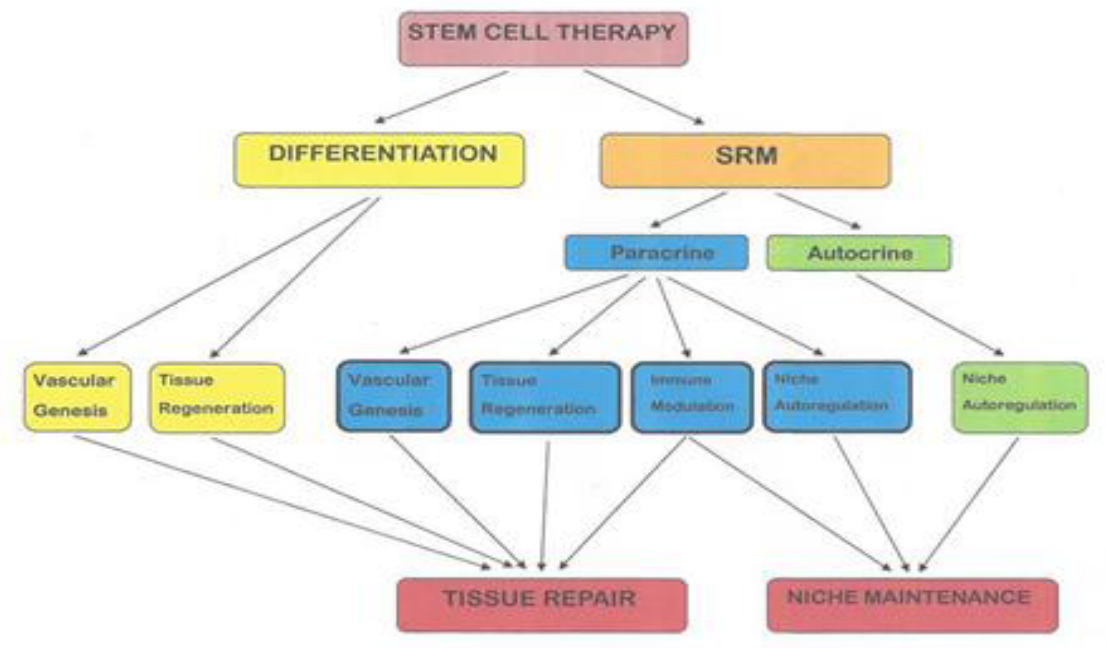

Figure 1: The actions of adult stem cells to achieve therapeutic values are carried out through two major pathways:

- $\quad$ Differentiation into new tissue, and

- Stem cell released molecules (SRM). Up to $80 \%$ of the healing actions of stem cells occur by way of SRM paracrine and autocrine mechanisms that are able to generate tissue, modulate the immune system and regulate the stem cell niche.

when compared to an isolated factor, is platelet derived growth factor (PDGF) [16]. SRM formulated to heal lower extremity diabetic ulcers has a significantly greater efficacy than a product (Regranex) which contains only one molecule (PDGF) $[4,16]$. Harnessing the power of stem cells promises to bring a systems approach to tissue repair, where a multitude of molecules are used to effectuate a system, or circuit, of mechanisms important for mimicking the healing properties inherent in the native tissue.

It has been demonstrated that two or more stem cell types can 'home' into damaged tissue, where each cell type releases a unique and often overlapping pool of SRM (labelled as S2RM) that act synergistically to induce healing (Figure 2). This S2RM technology represents a new, distinct direction in therapeutic development [4]. Reverse engineering technology led to the discovery of S2RM technology which mimics the natural stem cell healing processes. The multiple target pathways that underlie a particular indication can now be identified and perturbed for therapeutic molecular amelioration.

\section{Therapeutic Benefits of SRM-Based Products}

In 1957 stem cell therapeutics gained a major stake in medicine with the advent of intravenous infusion of bone marrow in cancer patients [23]. This led to today's use of purified stem cells rather than whole bone marrow taken from the patient before chemotherapy, thus avoiding the reintroduction to patients of their own cancer cells.

Academia and major medical teaching centres throughout the world have discovered an array of new therapeutic procedures involving stem cells, including: treatment for Type 1 diabetes [24]; bone tissue repair in osteogenesis imperfect [25]; promotion of tissue regeneration in myocardial infarction; and immune modulators in the treatment of graft-versus-host disease (GvHD) [26,27]. Organ transplant patients routinely benefit from stem cell transplantation: It resets the immune system, preventing organ rejection by replacing the deadly immunosuppressive drugs normally given to organ transplant patients [28].

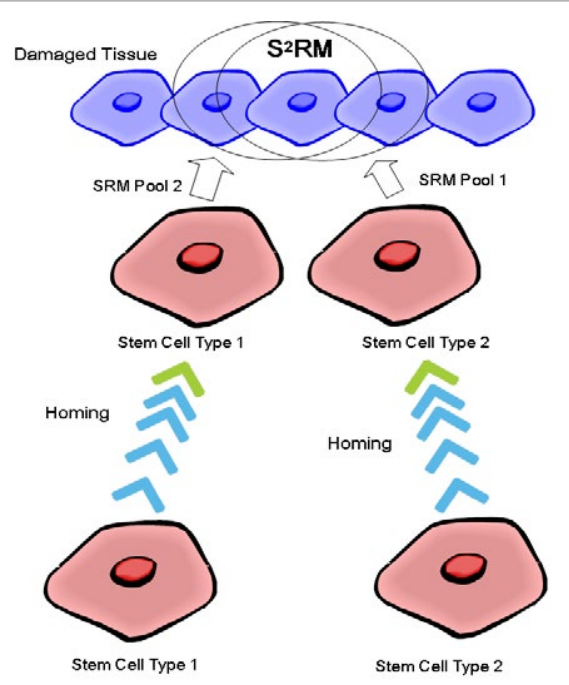

Figure 2: The synergistic combination of Pool 1 SRM + Pool 2 SRM, termed $\mathrm{S}^{2} \mathrm{RM}$, contains a fraction of exosomes.

Therapeutic SRM technologies include the use of SRM from a type of progenitor cell within an artificial skin matrix to close diabetic foot ulcers and S2RM in a number of other conditions [1,29].

Like the advent of gene-splicing in the 1970s and the 1976 delivery of human insulin as a commercially viable product that launched the biotechnology industry, the potential power of stem cell technology will impact on society for regeneration of cells and their therapy for decades. These scientific advancements are being experienced by humans every day. Interestingly, patients who are "pet parents" now are so aware of the benefits derived from stem cell science that they want their companion animals to receive similar treatments and medicines.

Current unmet, latent and evolving animal health care needs have fueled a demand for innovative technologies and therapies 
for pets. With all its potentials, it makes sense to extend the human applications and experiences with SRM technology to meet this demand. With the exception of direct stem cell injection into bones and joints to facilitate the healing of injuries of these tissues [12], as of yet no product utilizing SRM is commercially available to veterinary medicine. The opportunity to improve the overall health of companion animals through the application of topical, ingestible or injectable SRM based products remains unexplored.

\section{Application of SRM Technology to Companion Animals}

Stem cells are present in the tissues of all mammalian species. SRM are highly conserved communication tools: They convey functional information to the cells and tissues with which they come in contact, acting to induce tissue repair and regeneration, and modulation of the immune system. SRM is a bio-inspired system therapeutic that mimics the endogenous stem cell healing in the body. With up to $80 \%$ of the therapeutic function of adult stem cells in the body resulting from release of SRM, as opposed to differentiation of the stem cell into mature tissue, SRM technology can be readily integrated into a variety of treatments for all mammalian species, including companion animals.

Utilizing adult human stem cells as their SRM source, several topical products incorporating SRM technology have been developed for skin, hair and eye conditions in people. Already tested for safety and with proven efficacy, these products have significant therapeutic potentials in the treatment of parallel indications in canines. Examples discussed include allergic dermatitis, alopecia and keratitis sicca in dogs.

Canine atopic dermatitis (CAD) is a chronic, allergic skin disease that shares many similarities with atopic dermatitis and eczema in humans ${ }^{29}$. While there is utilization of SRM in skin care products developed and commercialized to improve the appearance and skin health for people, there have been no attempts to apply SRM-based products in veterinary medicine for this very common skin condition in animals.

The topical test product used contains the SRM technology for the delivery of key growth factors, anti-oxidants and other molecules found in natural human skin: TGF-b [transforming growth factor beta]; PDGF [platelet derived growth factor]; GM-CSF [granulocytemacrophage colony-stimulating factor]; and Interleukins (IL3, IL68), anti-oxidants and other protein molecules [1-3]. Along with a multitude of growth factors and other protein molecules, the highest concentration of antioxidants in the body is contained in the epidermis, the protective barrier for the living dermal layer and the internal body.

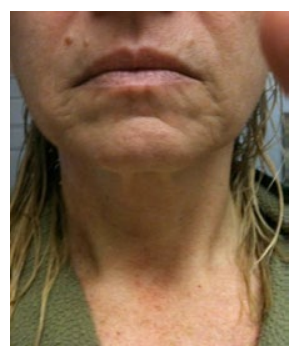

Before

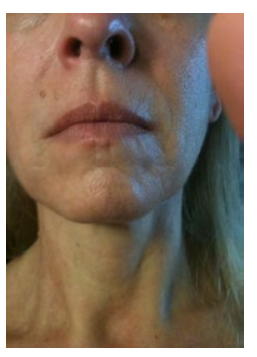

8 Weeks

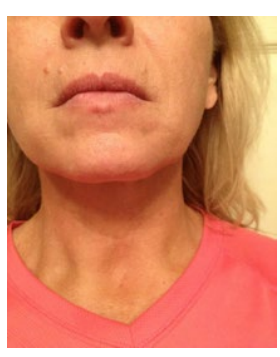

6 Months
Figure 3: Topical product exhibits improved quality and appearance of skin These images exemplify the ability of SRM technology to improve overall skin health.
With age, the concentration of growth factors and antioxidants in the skin declines considerably, and without its protective capacity, skin damage increases. Growth factors cannot be replaced in the skin through oral ingestion of antioxidants. But topically applied SRM growth factors and antioxidants can restore an effective antioxidant barrier, reducing further damage and allowing for regeneration of skin tissue.

\section{Canine Topical Application Pilot Study}

In a pilot study, an SRM-formulated topical skin application was applied to 40 dogs exhibiting a variety of skin conditions, including dry, atopic dermatitis and moist eczema ('hot spots'). A preliminary review indicated an overall reduction in skin irritation, with significant hot spot improvement over a two-week course of daily treatments.

The direct skin application of SRM-containing products, termed 'endogenous topical nutraceuticals' (ETN), can maintain and improve the overall health and condition of pets affected by allergic dermatitis, flea allergy dermatitis (FAD) and a host of common skin conditions.

While the effectiveness of topical SRM in people is typically measured in the reduction of 'fine lines and wrinkles', the functional outcome of ETN is an overall improvement in the health, vitality and fitness of the skin and a reduction in environmental dermal insult. ETN stimulates the underlying layers of the skin to build new collagen, fibronectin and elastin. It then orchestrates enhanced natural healing through antimicrobial activity of peptide LL37 and antifungals present in the SRM [25].

An SRM formulation similar to that used to treat the human alopecia has been developed and tested in dogs $(n=6)$ that presented with a variety of hair loss conditions. In all cases, hair regrowth began within two weeks of treatment initiation, with several owners reporting reduced shedding. Research has shown that SRM acts to nourish the hair follicle and mend the stem cell niche of the hair follicle (Figure 4).

\section{Keratitis Sicca Treatment}

Keratoconjunctivitis sicca (KCS, or 'dry eye syndrome') is characterized in both humans and canines by deficient tear production leading to severe drying and inflammation of the cornea and conjunctiva. A sterile, therapeutic ophthalmic drop was designed to treat KCS in humans, with an SRM composition of cultured stem cells that included growth factors, cytokines and additional elements supporting wound healing. The drops have since been shown to be

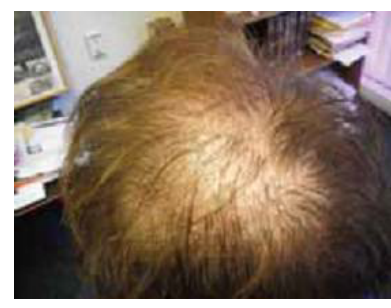

Before Treatment

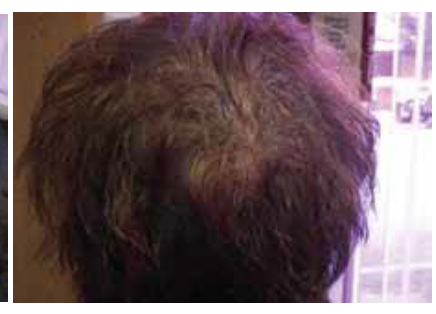

3 Months after Treatment
Figure 4: Alopecia treatment illustrates ETN capacity to regrow hair. After a one-week regimen of daily topical SRM applications, followed by applications every other day for the next 30 days, treatment was discontinued and hair allowed to grow in. 


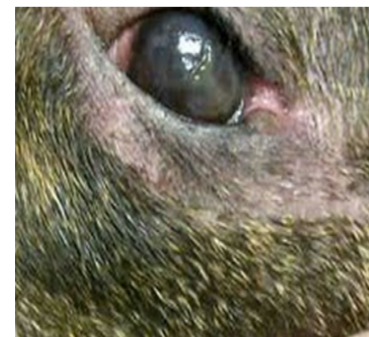

Day 0 Pretreatment

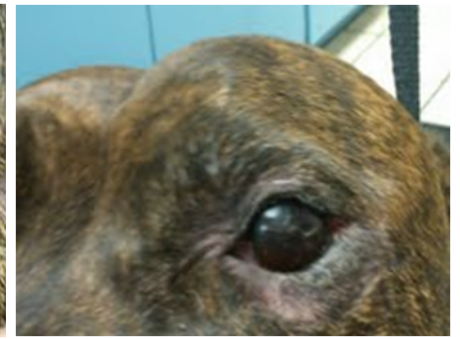

Day 7 after Treatment
Figure 5: Improvement seen in canine keratitis sicca after seven days of treatment.

safe and effective in patients presenting with dry eye, glaucoma and allergies. Human ophthalmologists reported success in $80+\%$ of patients using the treatment for KCS.

Brachiocephalic canine breeds, including pugs, Boston terriers and boxers, are particularly prone to dry eye syndrome. Dog breeders and professional handlers reported success rates averaging $70 \%$ in resolving dry eye in treated dogs (Figure 5).

\section{Conclusion}

SRM technology is based on the identification, selection, culture and stimulation of suitable stem cells to capture the molecules they release.

This systems-based approach to therapeutics mimics the body's normal stem cell cascade by utilizing a full complement of stem cell molecules. Injected or applied topically to a patient, they initiate tissue regeneration and healing. Having determined that humanderived SRM is both safe and effective for use in animals, additional therapeutic indications have been identified and are in various stages of research and development.

Given the significant, positive responses achieved when SRM is used as a topical product in dogs, a complete line of skin care SRMbased products will soon be commercially available as additional innovative treatment modalities targeting canine, feline and equine ophthalmology, dermatology and orthopedics.

\section{References}

1. Maguire G, Friedman P (2013) The systems biology of stem cell released molecules-based therapeutics. Int Sch Res Notices 2013: 12.

2. Maguire G, Friedman $P$ (2014). Enhancing spontaneous stem cell healing Journal of Complementary and Integrative Medicine. Biomed Rep 2: 163-166.

3. Chimenti I, Rachel RS, Tao-Sheng L, Gary G, Elisa M, et al. (2010). Relative roles of direct regeneration versus paracrine effects of human cardiospherederived cells transplanted into infarcted mice. Circ Res 106: 971-980.

4. Maguire G (2013) Using a systems-based approach for the development of diagnostics. Expert Review of Molecular Diagnostics.

5. Lai RC, Yeo RW, Tan KH, Lim SK (2012) Exosomes for drug delivery - a novel application for the mesenchymal stem cell. Biotecnol Adv 3:543-551.

6. Alvarez-Ervit L, Seow Y, Yin H, Betts C, Lakhal S, et al. (2011) Delivery of siRNA to the mouse brain by systemic injection of targeted exosomes. Nat Biotechnol 29: 341-345.

7. Mayshar Y, Ben-David U, Lavon N, Biancotti JC, Yakir B, et al. (2010) Identification and classification of chromosomal aberrations in human induced pluripotent stem cells. Cell Stem Cell 7: 521-531.

8. Lister R (2011) Hotspots of aberrant epigenomic reprogramming in human induced pluripotent stem cells. Nature 3: 68-73.
9. Scadden DT (2006) The stem cell niche as an entity of action. Nature 441 1075-1079.

10. Maniotis A, Chen CS, Ingber DE (1997) Demonstration of mechanical connections between integrins, cytoskeletal filaments, and nucleoplasm that stabilize nuclear structure. Proc Natl Acad Sci U S A. 4: 849-854.

11. Milstein JN, Meiners JC (2011) On the role of DNA biomechanics in the regulation of gene expression. J Roy Soc Interface 8: 1673-1681.

12. Sen B, Zhihui Xie, Natasha Case, Maya Styner, Clinton T Rubin, et al. (2010) Mechanical signal influence on mesenchymal stem cell fate is enhanced by incorporation of refractory periods into the loading regimen $\mathrm{J}$ Biomech 24: 593-599.

13. Engler AJ, Sen S, Sweeney HL, Discher DE (2006) Matrix Elasticity Directs Stem Cell Lineage Specification. Cell. 126: 677-689.

14. Discher DE, Mooney DJ, Zandstra $P$ (2009) Growth factors, matrices, and forces combine and control stem cells. Science 26: 1673-1677.

15. Viswanathan P, Somyot C, Kamolchanok N, Adam JE, Giuseppe B (2012) Cell Instructive Microporous Scaffolds through Interface Engineering. J Am Chem Soc 134: 20103-20109.

16. Thomas ED, Lochte HL Jr, Lu WC, Ferrebee JW (1957) Intravenous infusion of bone marrow in patients receiving radiation and chemotherapy. $\mathrm{N}$ Engl $\mathrm{J}$ Med 257: 491-496.

17. Zhao Y, Zhaoshun J, Tingbao Z, Mingliang Y, Chengjin H, et al. (2012) Reversal of type 1 diabetes via islet $\beta$ cell regeneration following immune modulation by cord blood-derived multipotent stem cells. BMC Med 10: 3 .

18. Mauney JR, Volloch V, Kaplan DL (2005) Role of adult mesenchymal stem cells in bone tissue engineering applications: current status and future prospects. Tissue Eng 11: 787-802.

19. Le Blanc K, Rasmusson I, Sundberg B, Götherström C, Hassan M, et al. (2004) Treatment of severe acute graft-versus-host disease with third party haploidentical mesenchymal stem cells. Lancet 363: 1439-1441.

20. Paul D, Samuel SM, Maulik N (2009) Mesenchymal stem cell: present challenges and prospective cellular cardiomyoplasty approaches for myocardial regeneration. Antioxid Redox Signal 11: 1841-1855.

21. Scandling JD, Busque S, Dejbakhsh-Jones S, Benike C, Sarwal M, et al. (2012) Tolerance and Withdrawal of Immunosuppressive Drugs in Patients Given Kidney and Hematopoietic Cell Transplants. Am J Transplant 12: 1133-1145.

22. Warriner RA, Cardinal M (2011) Human fibroblast-derived dermal substitute: results from a treatment investigational device exemption (TIDE) study in diabetic foot ulcers. Adv Skin Wound Care 24: 306-311.

23. Tao-Sheng L, Cheng K, Malliaras K, Smith RR, Zhang Y, et al. (2012) Direct comparison of different stem cell types and subpopulations reveals superior paracrine potency and myocardial repair efficacy with cardiosphere-derived cells. J Am Coll Cardiol. 59(10): 942-953.

24. Russo E (2003) Special Report: The Birth of Biotechnology. Nature 421: 456-457.

25. Black LL, Gaynor J, Gahring D, Adams C, Aron D, et al. (2007) Effect of Adipose Derived Mesenchymal Stem Cells and Regenerative Cells on Lameness in Dogs. Veterinarian Therapeutics 8.

26. Baillie JK, Barnett MW, Upton KR, Gerhardt DJ, Richmond TA, et al. (2011) Somatic retro- transposition alters the genetic landscape of the human brain. Nature 479: 534-537.

27. Kaiser J (2012) Genetic influences on disease remain hidden," Science 338 1016-1017.

28. Magi A, Tattini L, Benelli M, Giusti B, Abbate R, et al. (2012) WNP: a novel algorithm for gene products annotation from weighted functional networks," PLOS ONE 7: e38767.

29. Nødtvedt, Ane (2007) Epidemiology of canine atopic dermatitis. Diss. (sammanfattning/summary) Uppsala: Sveriges lantbruksuniv., Acta Universitatis agriculturae Sueciae 47: 1652-6880.

\section{Author Affiliation}

${ }^{1}$ D. V.M., M.R.C.V.S. TWH Consulting, LLC, Midlothian, Virginia, USA

${ }^{2}$ B.A. President, Animal Biosciences, Helen Circle, Bartow, Florida, USA

${ }^{3}$ B.S. Chem. Vice President, Animal Biosciences, Orange Park, Florida, USA 